\title{
Different effects of monophasic pulses and biphasic pulses applied by a bipolar stimulation electrode in the rat hippocampal CA1 region
}

Yue Yuan, Lvpiao Zheng, Zhouyan Feng ${ }^{*}$ (D) and Gangsheng Yang

${ }^{*}$ Correspondence:

fengzhouyan@zju.edu.cn

Key Laboratory of Biomedical Engineering of Education

Ministry, College

of Biomedical Engineering

and Instrumentation

Science, Zhejiang University,

Hangzhou 310027, Zhejiang,

China

\begin{abstract}
Background: Electrical pulse stimulations have been applied in brain for treating certain diseases such as movement disorders. High-frequency stimulations (HFS) of biphasic pulses have been used in clinic stimulations, such as deep brain stimulation (DBS), to minimize the risk of tissue damages caused by the electrical stimulations. However, HFS sequences of monophasic pulses have often been used in animal experiments for studying neuronal responses to the stimulations. It is not clear yet what the differences of the neuronal responses to the HFS of monophasic pulses from the HFS of biphasic pulses are.
\end{abstract}

Methods: To investigate the neuronal responses to the two types of pulses, orthodromic-HFS (O-HFS) and antidromic-HFS (A-HFS) of biphasic and monophasic pulses (1-min) were delivered by bipolar electrodes, respectively, to the Schaffer collaterals (i.e., afferent fibers) and the alveus fibers (i.e., efferent fibers) of the rat hippocampal CA1 region in vivo. Evoked population spikes of CA1 pyramidal neurons to the HFSs were recorded in the CA1 region. In addition, single pulses of antidromic- and orthodromic-test stimuli were applied before and after HFSs to evaluate the baseline and the recovery of neuronal activity, respectively.

Results: Spreading depression (SD) appeared during sequences of 200-Hz monophasic O-HFS with a high incidence (4/5), but did not appear during corresponding $200-\mathrm{Hz}$ biphasic O-HFS (0/6). A preceding burst of population spikes appeared before the SD waveforms. Then, the SD propagated slowly, silenced neuronal firing temporarily and resulted in partial recovery of orthodromically evoked population spikes (OPS) after the end of O-HFS. No SD events appeared during the O-HFS with a lower frequency of $100 \mathrm{~Hz}$ of monophasic or biphasic pulses (0/5 and 0/6, respectively), neither during the A-HFS of $200-H z$ pulses (0/9). The antidromically evoked population spikes (APS) after 200-Hz biphasic A-HFS recovered to baseline level within 2 min. However, the APS only recovered partially after the 200-Hz A-HFS of monophasic pulses.

Conclusions: The O-HFS with a higher frequency of monophasic pulses can induce the abnormal neuron activity of SD and the A-HFS of monophasic pulses can cause a persisting attenuation of neuronal excitability, indicating neuronal damages caused

(c) The Author(s) 2021. This article is licensed under a Creative Commons Attribution 4.0 International License, which permits use, sharing, adaptation, distribution and reproduction in any medium or format, as long as you give appropriate credit to the original author(s) and the source, provide a link to the Creative Commons licence, and indicate if changes were made. The images or other third party material in this article are included in the article's Creative Commons licence, unless indicated otherwise in a credit line to the material. If material is not included in the article's Creative Commons licence and your intended use is not permitted by statutory regulation or exceeds the permitted use, you will need to obtain permission directly from the copyright holder. To view a copy of this licence, visit http://creativecommons.org/ licenses/by/4.0/. The Creative Commons Public Domain Dedication waiver (http://creativecommons.org/publicdomain/zero/1.0/) applies to the data made available in this article, unless otherwise stated in a credit line to the data. 
by monophasic stimulations in brain tissues. The results provide guidance for proper stimulation protocols in clinic and animal experiments.

Keywords: High-frequency stimulation, Monophasic pulse, Biphasic pulse, Spreading depression, Hippocampal CA1 region

\section{Background}

Electrical pulses have been used in deep brain stimulation (DBS) for treating certain neurological disorders successfully (e.g., Parkinson's disease and epilepsy) and for treating psychiatric disorders promisingly (e.g., major depression and obsessive compulsive disorder) $[1,2]$. The DBS commonly utilizes sequences of narrow pulses with a pulse width around $0.1 \mathrm{~ms}$ and a pulse frequency $\sim 100-200 \mathrm{~Hz}$ (termed as high-frequency stimulation, HFS). Charge-balanced biphasic pulses (with a preceding negative pulse immediately followed by a positive one) are usually utilized in clinical DBS for safety [3, 4], while negative monophasic pulses are used in animal studies for investigating brain stimulations [5-7]. However, it is not clear whether HFS sequences of monophasic pulses could induce abnormal neuronal responses different from biphasic pulses.

In theory, a negative pulse is more efficient to activate neurons than a positive pulse or a biphasic pulse in the situation of extracellular stimulation such as DBS $[8,9]$. However, a continuous stimulation of a sequence of monophasic pulses could risk tissue damages caused by irreversible chemical reactions, whereas biphasic pulses could not $[8,10]$. The reverse electric field generated by the subsequent positive phase of a biphasic pulse may prevent an accumulation of cation/anion ions thereby preventing irreversible chemical reactions in the brain tissue around the electrode contacts. However, sequences of monophasic negative pulses could result in irreversible chemical reactions to generate toxic products to damage the brain tissue [8].

Besides the toxic products of electrochemical reactions, stimulation-related tissue damages may be also caused by other factors, such as depletion of oxygen by hyperactivity of neuronal firing, substantial changes of ionic concentrations in both intracellular and extracellular environments, and excitotoxicity caused by excessive release of glutamates $[8,11]$. Some of the changes may lead to abnormal neuronal activities, such as a large shift of depolarization potential in brain regions, termed as spreading depression (SD) [12]. To investigate the differences of neuronal responses to HFS of monophasic pulses and biphasic pulses, we recorded the electrical signals in the hippocampal CA1 region in rat brain in vivo during HFS of axonal fibers and observed the neuronal responses.

The hippocampal region of brain is prone to generate SD events under certain circumstances, such as an increase of extracellular potassium [13, 14]. In addition, axons are the most susceptible structure of neurons to the extracellular stimulation of narrow electrical pulses $[15,16]$. Therefore, the incidence of SD induced by axonal HFS in hippocampal CA1 region could be used as a biomarker to distinguish the neuronal responses to different sequences of HFS. Results of the study may provide new information for appropriate usage of pulse stimulations in the investigations and in the clinical applications of brain stimulations. 


\section{Results}

\section{Spreading depression appeared during orthodromic-HFS with monophasic} but not biphasic pulses

To investigate the different effects between biphasic and monophasic pulses, HFS sequences of pulses were delivered through the stimulation electrodes placed at the Schaffer collaterals for the orthodromic-HFS (O-HFS) and at the alveus fibers for the antidromic-HFS (A-HFS), respectively (Fig. 1a). The stimulation electrodes were a concentric bipolar structure (Fig. 1b). For the stimulations of a single pulse with an identical current intensity, the mean amplitude of orthodromically evoked population spike (OPS) induced by a monophasic pulse $(8.62 \pm 1.97 \mathrm{mV}, n=5)$ was not significantly different from that induced by a biphasic pulse $(8.40 \pm 2.16 \mathrm{mV}, n=6$; $t$-test, $P=0.95$. Fig. $1 \mathrm{c}$ and $\mathrm{d}$ ), which indicated a similar excitation action of the two types of pulses.

During the O-HFS of 1-min $200 \mathrm{~Hz}$ of biphasic pulses, OPS events only appeared in the initial several seconds of O-HFS (Fig. 2a). After the disappearance of OPS, multiple unit activity (MUA) continued to the end of the O-HFS with a firing rate of unit spikes higher than baseline. A silent period (10-30 s) without MUA appeared immediately following the end of O-HFS, indicating that the unit spikes during the late O-HFS period were induced by the stimulation. During the period of O-HFS, an antidromic-test (A-test) pulse was applied every $5 \mathrm{~s}$ (i.e., $0.2 \mathrm{~Hz}$ ) at the alveus fibers to evaluate the excitability of the CA1 neurons. The single A-test pulses and orthodromic-test (O-test) pulses were also applied before and after O-HFS to evaluate the baseline and the recovery state

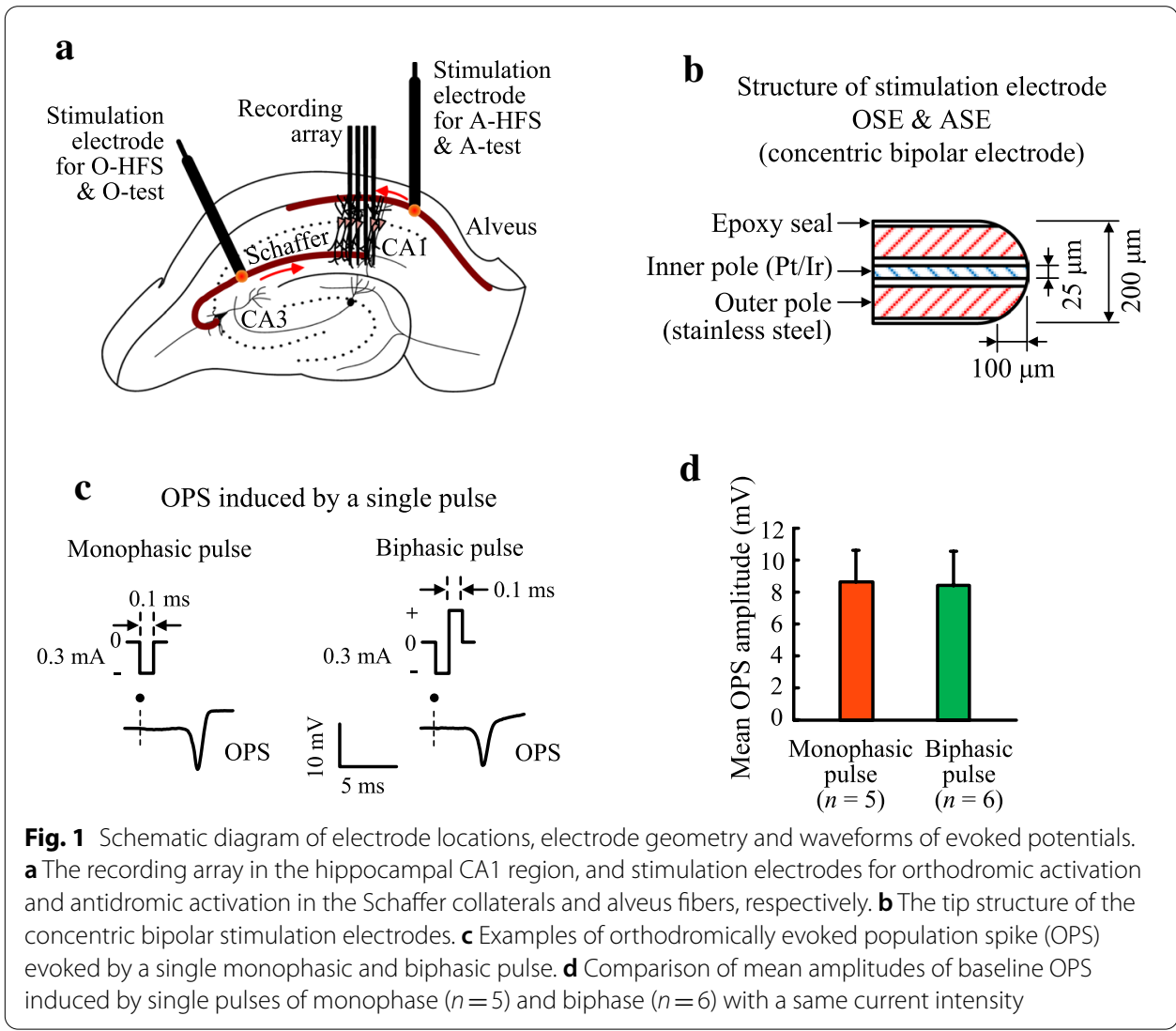




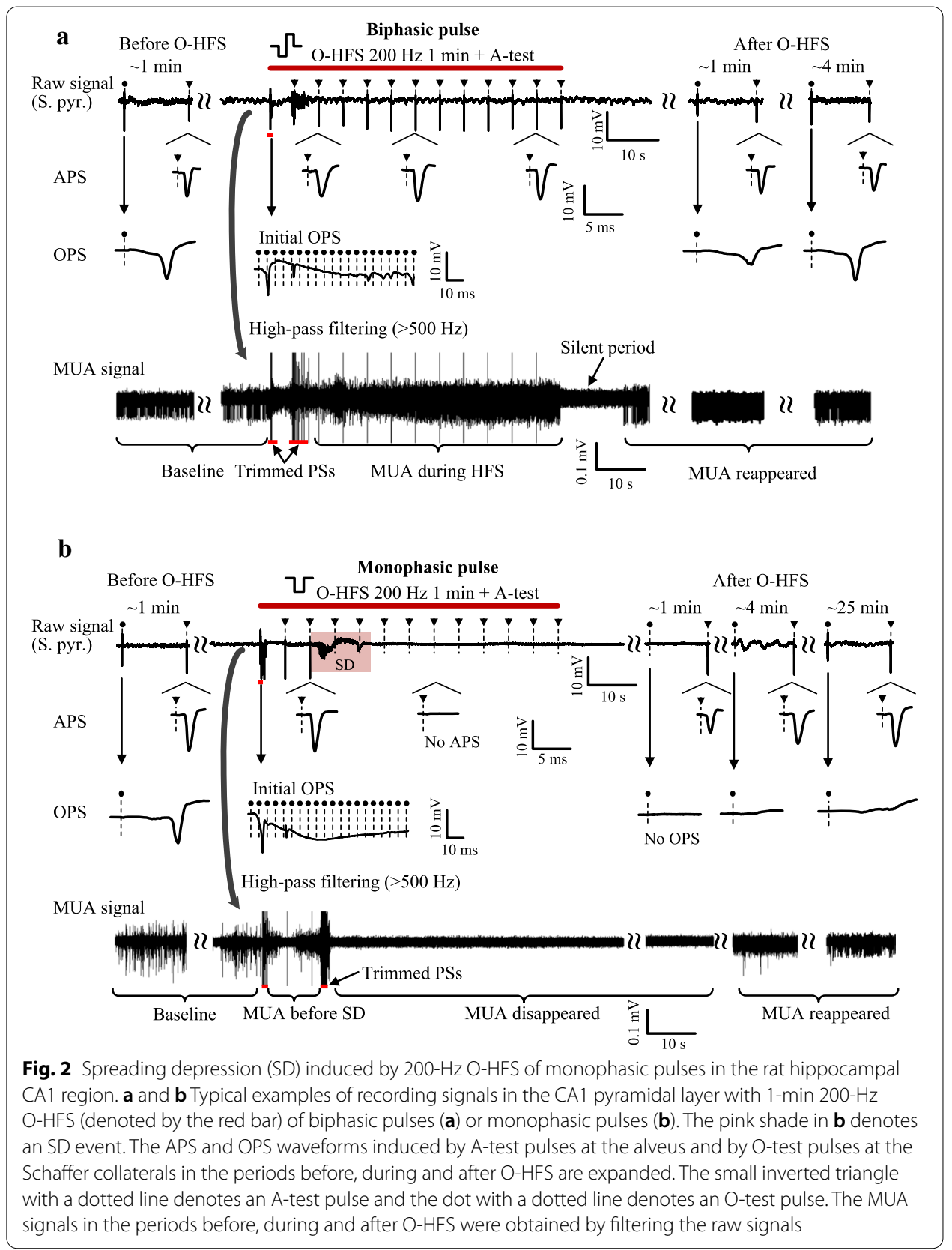

of neuronal activity, respectively. Large antidromically evoked population spike (APS) evoked by A-test pulses persisted throughout the 1-min O-HFS, and the mean amplitude of these APS $(7.26 \pm 5.59 \mathrm{mV}, n=6)$ was $\sim 17 \%$ greater than the corresponding baseline level $(6.19 \pm 3.19 \mathrm{mV}, n=6$; paired $t$-test, $P<0.05)$. The results indicated that the sustained O-HFS increased the excitability of the CA1 neurons. About 4 min after the end of O-HFS, both test APS and test OPS evoked by single pulses recovered to the baseline level. In addition, no SD event appeared in all of the 6 rats with the $200-\mathrm{Hz}$ biphasic O-HFS.

However, SD events appeared in 4 of the 5 rats with the 200-Hz monophasic O-HFS (Fig. 2b). The initial neuronal responses induced by the monophasic O-HFS was similar to 


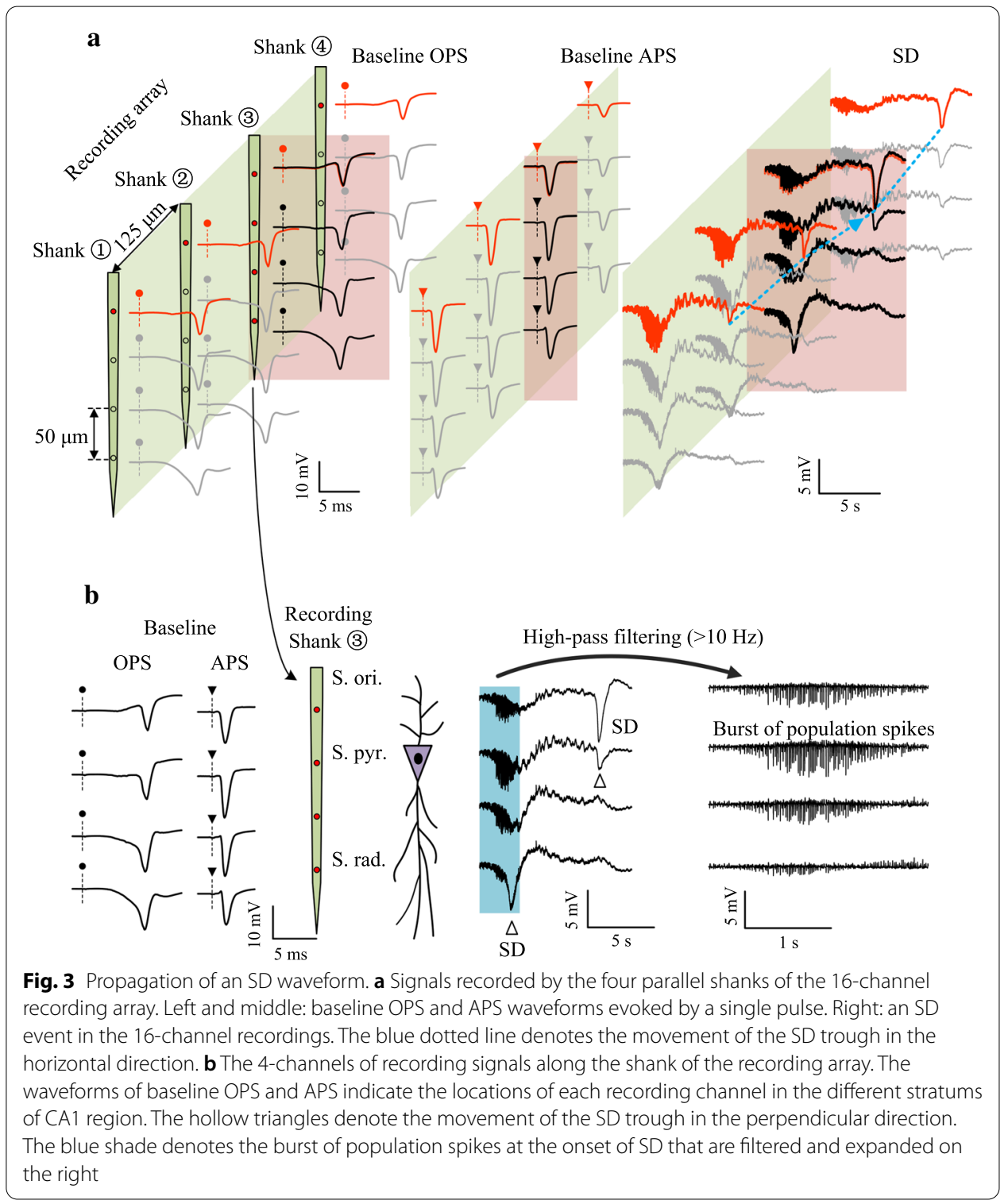

that induced by the biphasic O-HFS: large OPS appeared at first, then OPS disappeared and dense MUA appeared. However, an SD appeared later with a slow waveform lasting $4.31 \pm 3.11 \mathrm{~s}(n=4)$. At the same time, the MUA disappeared completely and the A-test pulses were no longer able to induce an APS, indicating a silence of neuronal activity. The MUA did not appear until $3.51 \pm 2.47 \mathrm{~min}(n=4)$ after the end of O-HFS (Fig. 2b, bottom). By this time, the amplitude of test APS recovered to 80\% of the baseline level. However, the test OPS did not recover even $\sim 25 \mathrm{~min}$ after the end of O-HFS, while the test APS had almost recovered to baseline level $(89.5 \pm 9.7 \%, n=4$. Fig. $2 \mathrm{~b}$, middle). The results indicated that 1-min persisted $200 \mathrm{~Hz}$ of monophasic O-HFS generated abnormal reactions of the neuronal population.

To show the spread of SD waveforms, we used the recording electrode array with four shanks and total 16 contacts (Figs. 1a and 3a). The waveforms of OPS and APS along the 
shanks in baseline recordings indicated the locations of each recording contact in the different stratums of CA1 region [17]. Because the signal recordings in the study were ACcoupled $(0.3-5000 \mathrm{~Hz})$, the SD waveform appeared as a trough similar to previous reports [18]. The SD trough appeared first in the stratum radiatum (S. rad.) of hippocampal CA1 region (Fig. 3b) and accompanied by a burst of population spikes (60-80 spikes/s) in the stratum pyramidale (S. pyr.). The burst was obvious in the filtered signals greater than $10 \mathrm{~Hz}$ (Fig. 3b right). Then, the SD trough propagated slowly to the CA1 layers of S. pyr. and stratum oriens (S. ori.) at a speed of $90 \pm 51 \mu \mathrm{m} / \mathrm{s}(n=4)$ in the perpendicular direction, characterized by the movement of the negative peak of SD trough along the recording shanks (Fig. 3b, hollow triangles). Also, the SD trough moved at a speed of $826 \pm 627 \mu \mathrm{m} / \mathrm{s}$ $(n=4)$ in the S. pyr. layer transversely among the recording shanks (Fig. 3a right, blue dotted line). The characteristics of the SD events, including the waveform, the accompanied burst of population spikes, the slow travelling speed and the silence of neuronal electrical activity, were consistent with previous reports $[18,19]$.

The statistical results showed that the SD incidence during 200-Hz monophasic O-HFS (4/5) was significantly greater than the incidence during $200-\mathrm{Hz}$ biphasic O-HFS (0/6; Fisher's exact test, $P<0.05)$. In addition, with a decrease of the O-HFS frequency from 200 to $100 \mathrm{~Hz}$, no SD events were observed with monophasic O-HFS (five rats) or with biphasic O-HFS (six rats). Therefore, for the data of monophasic O-HFS only, the SD incidence during $100-\mathrm{Hz}$ O-HFS $(0 / 5)$ was significantly lower than that during $200-\mathrm{Hz} \mathrm{O}-\mathrm{HFS}(4 / 5$; Fisher's exact test, $P<0.05)$.

These results indicated that O-HFS of monophasic pulses with a higher stimulation frequency can generate SD events in the hippocampal CA1 region and affect the orthodromic pathway persistently. Because the generation of OPS by the stimulation at afferent fibers involves both the axonal conductions and the synaptic transmissions, the non-recovery of OPS after the monophasic O-HFS could have been caused by potential damages in the axons and/or synapses. To verify whether the monophasic HFS could cause damages in axons, we next inspected the responses of CA1 neurons to the A-HFS at their own axons (i.e., the alveus) without involving synaptic transmissions.

\section{No spreading depression but more attenuation of APS amplitudes caused by A-HFS of monophasic pulses}

To investigate the neuronal responses to the A-HFS of biphasic and monophasic pulses, we analyzed each APS evoked by each pulse of A-HFS. During the 1-min 200-Hz A-HFS of biphasic pulses, APS was able to follow each stimulation pulse. However, the APS waveforms with a large amplitude only appeared at the initial period. The APS amplitudes decreased rapidly with the proceeding of A-HFS (Fig. 4a), which may be caused by the depolarization block of axons [5, 20]. After the end of A-HFS, the test APS recovered to $\sim 70 \%$ of baseline level in $\sim 1 \mathrm{~min}$ and to $92.2 \pm 21.0 \%(n=4)$ of baseline level in $\sim 2$ min.

When the A-HFS was applied with monophasic pulses, the neuronal responses at the initial period were similar to that of biphasic pulses-each pulse induced a large APS. However, at the late period of A-HFS, the monophasic pulses hardly induced APS (Fig. 4b). In addition, after the end of A-HFS, the test APS only recovered to $34.5 \pm 12.1 \%$ $(n=5)$ of baseline level even after $\sim 20 \mathrm{~min}$. 


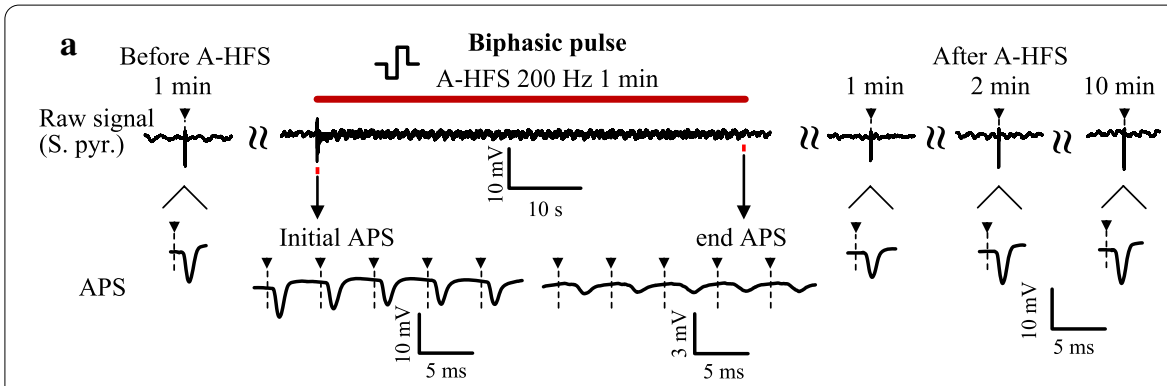

b
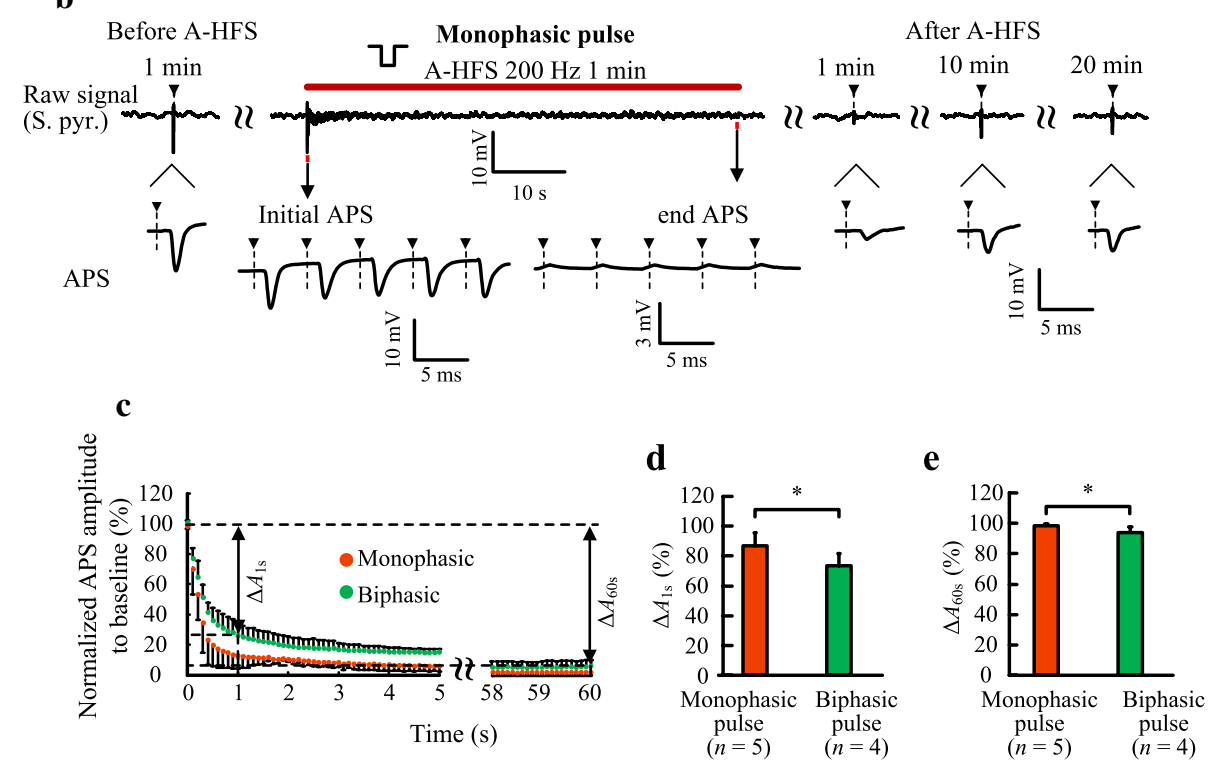

Fig. 4 Neuronal responses induced by 200-Hz A-HFS in the hippocampal CA1 region. $\mathbf{a}$ and b Top: typical examples of recording signals in the S. pyr. with 1-min 200-Hz A-HFS (denoted by the red bar) of biphasic pulses (a) or monophasic pulses (b). Bottom: typical waveforms of APS induced by A-test pulses or A-HFS pulses in the periods before, during and after A-HFS. c Normalized APS amplitude in the first $5 \mathrm{~s}$ and last $2 \mathrm{~s}$ of 1-min A-HFS. $\mathbf{d}$ Comparison of the decrement of APS amplitude at $1 \mathrm{~s}\left(\triangle A_{1 s}\right)$ between monophasic A-HFS $(n=5)$ and biphasic A-HFS $(n=4)$. e Comparison of the decrement of APS amplitude at $60 \mathrm{~s}\left(\triangle A_{60 \mathrm{~s}}\right)$ between monophasic A-HFS $(n=5)$ and biphasic A-HFS $(n=4) .{ }^{*} P<0.05$, $t$-test

To compare the attenuations of APS during the A-HFS with biphasic and monophasic pulses, we calculated the APS amplitudes normalized to baseline value to evaluate the changes of APS (Fig. 4c). The decrement of APS amplitudes at the initial $1 \mathrm{~s}\left(\Delta A_{1 \mathrm{~s}}\right)$ of A-HFS was significantly greater with monophasic A-HFS $(86.9 \pm 8.7 \%, n=5)$ than with biphasic A-HFS ( $73.5 \pm 8.0 \%, n=4 ; t$-test, $P<0.05$. Fig. $4 \mathrm{~d})$ ). Also, the decrement of APS amplitudes at the end of A-HFS $\left(\triangle A_{60 \mathrm{~s}}\right)$ was significantly greater with monophasic A-HFS $(98.6 \pm 1.2 \%, n=5)$ than with biphasic A-HFS $(94.1 \pm 3.4 \%, n=4$; $t$-test, $P<0.05$. Fig. 4e).

The faster and larger attenuation of APS amplitudes during monophasic A-HFS indicated that the monophasic pulses can cause more conduction failures than biphasic pulses. The partial recovery of test APS after monophasic A-HFS indicated that the conduction failures in at least a portion of axons can even persist after the A-HFS. 
In addition, no SD event was observed in all the 9 rats applied 1-min 200-Hz A-HFS (4 with biphasic pulses and 5 with monophasic pulses). That is, the SD incidence during $200-\mathrm{Hz}$ monophasic A-HFS (0/5) was significantly smaller than that during $200-\mathrm{Hz}$ monophasic O-HFS (4/5; Fisher's exact test, $P<0.05)$.

\section{Discussion}

The major findings of the present study in the rat hippocampal CA1 region include: $\mathrm{O}-\mathrm{HFS}$ of monophasic pulses at the afferent fibers with an enough high frequency can induce spreading depression (SD) events and affect the pathway of orthodromic activation; and A-HFS of monophasic pulses at the efferent fibers can cause conduction failures in a portion of axons even after the A-HFS. Possible mechanisms underlying the findings are discussed below.

SD is a type of large shift of depolarization potential in brain regions. The earliest discovery of SD was associated with epileptic bursts and was described as a suppression of electroencephalographic signals following bursts of seizure discharges in the cortex regions [21]. Studies have also shown that SD events can follow neuronal discharges in other brain regions, such as in hippocampus [18]. One of the major associated characteristics of SD is the great concentration changes of certain ions in the extracellular space, especially a substantial elevation of extracellular potassium concentration $\left(\left[\mathrm{K}^{+}\right]_{\mathrm{o}}\right)$, indicating redistributions of the ions between extra- and intra-cellular spaces [22].

In the present study, SD events only appeared during monophasic O-HFS but not during biphasic O-HFS. Thus, certain effects associated with the HFS of monophasic pulses could be responsible for the appearance of SD. In contrary to the biphasic pulses, the monophasic pulses drive the ions in fixed directions in the brain tissue by the force of the unidirectional electric field. The cations migrate to the cathodic pole of the electrode and the anions to the anodic one. Under the action of repetitive monophasic pulses with a high frequency, the ions may accumulate around the two poles of the electrode and destroy the ionic equilibriums between the intracellular and extracellular environments. Previous studies have shown that repetitive stimulations can enhance $\left[\mathrm{K}^{+}\right]_{\mathrm{o}}$ in the CA1 region [23]. Presumably, the increase of $\left[\mathrm{K}^{+}\right]_{0}$ may be exacerbated by the HFS of monophasic pulses because of the cationic accumulation by the unidirectional driving force around the small space of electrode poles (Fig. 1b). The high $\left[\mathrm{K}^{+}\right]_{\mathrm{o}}$ may result in a depolarization in neuronal membranes and generate epileptiform activity [24, 25], just as the burst of population spikes preceding SD trough in the present study (Fig. 3b). The burst activity may further elevate $\left[\mathrm{K}^{+}\right]_{0}$ thereby resulting in the large shift of depolarization potential of SD. Given the fact that a potassium injection with a molar level of concentration can induce SD events in animal preparations [14, 26, 27], the accumulation of $\mathrm{K}^{+}$ by monophasic pulses may be one of the mechanisms to generate SD.

In addition, synapse-related effects could also contribute to the generation of SD, because SD events only appeared during O-HFS involving synaptic transmissions but did not appear during A-HFS. Previous studies have shown that an excessive concentration of glutamates released from excitatory synapses to the extracellular space can induce SD [27-29]. In the present study, the O-HFS in the afferent fibers can activate the excitatory synapses and can cause glutamates releases at the layer of apical dendrites 
of pyramidal neurons (i.e., S. rad.) that is full of glutamatergic synapses. In addition, the elevation of $\left[\mathrm{K}^{+}\right]_{\mathrm{o}}$ by monophasic O-HFS may reduce the uptake of glutamates and reduce extracellular space by astrocytes swelling, thereby further increasing the concentration of extracellular glutamates [30, 31]. Thus, an elevation of glutamates caused by monophasic O-HFS can be another factor to generate SD.

Furthermore, the non-recovery of the test OPS after monophasic O-HFS and the partial recovery of APS after monophasic A-HFS suggested that the application of intensive monophasic pulses could have damaged the axons under the stimulations. As previously reported, the toxic chemical products of irreversible chemical reactions at the electrode-tissue interface generated by monophasic stimulations can damage the neuronal tissues [8]. The reactions include electrolysis of water, oxidation of saline, metal, and organic materials, as well as reduction of oxygen [32, 33]. In addition, according to mass action theory, an extreme excitation by over-stimulating the excitable tissue can result in the depletion of oxygen or/and glucose thereby substantially changing the ionic concentrations in both intracellular and extracellular regions [8]. These factors may result in neuronal dysfunction and tissue damages. Further histological and morphological analyses of stimulated tissues are needed to confirm the tissue damages. Nevertheless, the appearance of SD can act as an index to warn the tissue damages.

Taken together, an extreme elevation of $\left[\mathrm{K}^{+}\right]_{\mathrm{o}}$ and an increase of glutamate concentrations can result in the generation of SD by the monophasic O-HFS. In addition, the irreversible chemical reactions induced by the monophasic HFS with an enough high frequency could damage a portion of the axons in the stimulation site.

\section{Conclusions}

Our results showed that orthodromically activating hippocampal neurons by HFS of monophasic pulses can induce the abnormal neuron activity of spreading depression, and antidromic activation can cause an attenuation of neuronal excitability persisting after HFS stimulations. The results suggest that HFS of monophasic pulses have a risk of tissue damage and can induce neuronal reactions different from HFS of biphasic pulses, which provide new information for appropriate usage of pulse stimulations in brain tissues either in clinic or in animal experiments.

\section{Methods}

\section{Surgical procedures}

The animal experiment was approved by the Institutional Animal Care and Use Committee, Zhejiang University. Twenty adult male Sprague-Dawley rats were used: 11 for O-HFS ( 6 for biphasic pulses and 5 for monophasic pulses) and 9 for A-HFS (4 for biphasic pulses and 5 for monophasic pulses). Each rat was anesthetized with urethane $(1.25 \mathrm{~g} / \mathrm{kg}$, i.p.) and was placed in a stereotaxic apparatus (Stoelting Co.). Details of the surgery and the electrode placements were similar to the previous report [34]. Briefly, a 16-channel recording electrode array (\#A4 $\times 4$-3 mm 50-125-177, NeuroNexus Technologies, USA) was inserted into the hippocampal CA1 region to record electrical potentials. Two concentric bipolar stimulation electrodes (\#CBBRC75, FHC Inc., USA) were positioned in the Schaffer collaterals and the alveus fibers of CA1 region 
for orthodromic- and antidromic-HFS, respectively (Fig. 1a). The distance between the inner and outer poles of the stimulation electrodes was short $(<100 \mu \mathrm{m})(\mathrm{Fig} .1 \mathrm{~b})$, indicating a small area around the two poles. The signals of multiple unit activity (MUA) and the waveforms of both orthodromically evoked population spike (OPS) and antidromically evoked population spike (APS) along the recording channels were used to guide the final positions of the three electrodes.

\section{Stimulation and recording}

Sequences of current pulses of monophase (negative phase) and biphase (a preceding negative phase followed by a positive phase) with a phase width of $0.1 \mathrm{~ms}$ (Fig. 1c) were generated by a stimulator (Model 3800, A-M Systems Inc., USA). The current intensity was $0.3-0.5 \mathrm{~mA}$ that was able to induce a population spike (PS) with an amplitude approximately $75 \%$ of the maximal PS amplitude. The pulse frequency was 100 or $200 \mathrm{~Hz}$ for O-HFS at the Schaffer collaterals, and $200 \mathrm{~Hz}$ for A-HFS at the alveus fibers of the hippocampal CA1 region. The duration was $1 \mathrm{~min}$ for both O- and A-HFS.

Recording signals were amplified 100 times by an AC-coupled $(0.3-5000 \mathrm{~Hz})$ amplifier (Model 3600, A-M Systems Inc., USA). The amplified signals were then sampled at a rate of $20 \mathrm{kHz}$ by a Powerlab data acquisition system (ADInstruments Inc., Australia).

\section{Data analysis}

The artifacts of stimulation pulses in the raw recording signals were removed by an algorithm to detect each artifact segment $(\sim 1.0 \mathrm{~ms})$ that was then replaced by an interpolation line [35]. The artifact-free signals were then filtered by high-pass digital filters with a cut-off frequency of $500 \mathrm{~Hz}$ to obtain the MUA signals, or with a cut-off frequency of $10 \mathrm{~Hz}$ to remove the slow waveform of SD to illustrate the burst population spikes accompanying SD. The recording signals from the channel closest to the cell body layer (i.e., S. pyr.) were used to calculate the amplitudes of evoked-PS.

Data were expressed as mean \pm standard deviation and $n$ represented the number of rat experiments. Statistical Fisher's exact test and $t$-test were used to judge the significance of differences among data groups.

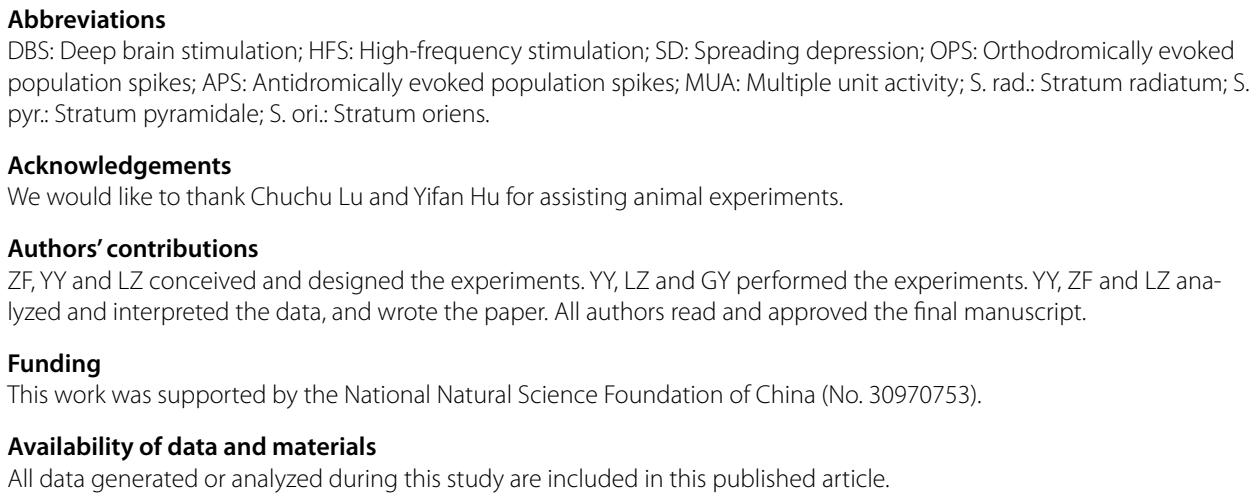




\section{Declarations}

\section{Ethics approval and consent to participate}

This study was carried out in accordance with the recommendations of Guide for the Care and Use of Laboratory Animals by China Ministry of Health. The protocol was approved by the Institutional Animal Care and Use Committee, Zhejiang University, Hangzhou.

\section{Consent for publication}

Not applicable.

\section{Competing interests}

The authors declare that they have no competing interests.

Received: 4 July 2020 Accepted: 1 March 2021

Published online: 09 March 2021

\section{References}

1. Lozano AM, Lipsman N, Bergman H, Brown P, Chabardes S, Chang JW, et al. Deep brain stimulation: current challenges and future directions. Nat Rev Neurol. 2019;15(3):148-60. https://doi.org/10.1038/s41582-018-0128-2.

2. Cagnan H, Denison T, McIntyre C, Brown P. Emerging technologies for improved deep brain stimulation. Nat Biotechnol. 2019;37(9):1024-33. https://doi.org/10.1038/s41587-019-0244-6.

3. Almeida L, Martinez-Ramirez D, Ahmed B, Deeb W, Jesus S, Skinner J, et al. A pilot trial of square biphasic pulse deep brain stimulation for dystonia: the BIP dystonia study. Mov Disord. 2017;32(4):615-8. https://doi.org/10.1002/ mds.26906.

4. De Jesus S, Okun MS, Foote KD, Martinez-Ramirez D, Roper JA, Hass CJ, et al. Square biphasic pulse deep brain stimulation for parkinson's disease: the BiP-PD study. Front Hum Neurosci. 2019. https://doi.org/10.3389/fnhum 2019.00368 .

5. Jensen AL, Durand DM. High frequency stimulation can block axonal conduction. Exp Neurol. 2009;220(1):57-70. https://doi.org/10.1016/j.expneurol.2009.07.023.

6. Fattahi M, Ashabi G, Karimian SM, Riahi E. Preventing morphine reinforcement with high-frequency deep brain stimulation of the lateral hypothalamic area. Addict Biol. 2018;24(4):685-95. https://doi.org/10.1111/adb.12634.

7. Feddersen B, Vercueil L, Noachtar S, David O, Depaulis A, Deransart C. Controlling seizures is not controlling epilepsy: a parametric study of deep brain stimulation for epilepsy. Neurobiol Dis. 2007;27(3):292-300. https://doi. org/10.1016/j.nbd.2007.05.005.

8. Merrill DR, Bikson M, Jefferys JGR. Electrical stimulation of excitable tissue: design of efficacious and safe protocols. J Neurosci Meth. 2005;141(2):171-98. https://doi.org/10.1016/j.jneumeth.2004.10.020.

9. Weitz AC, Behrend MR, Ahuja AK, Christopher P, Wei J, Wuyyuru V, et al. Interphase gap as a means to reduce electrical stimulation thresholds for epiretinal prostheses. J Neural Eng. 2014;11(1):16007. https://doi.org/10.1088/17412560/11/1/016007.

10. Piallat B, Chabardès S, Devergnas A, Torres N, Allain M, Barrat E, et al. Monophasic but not biphasic pulses induce brain tissue damage during monopolar high-frequency deep brain stimulation. Neurosurgery. 2009;64(1):156-62. https://doi.org/10.1227/01.neu.0000336331.88559.cf.

11. Butterwick A, Vankov A, Huie P, Freyvert Y, Palanker D. Tissue damage by pulsed electrical stimulation. IEEE T Bio-Med Eng. 2007;54(12):2261-7. https://doi.org/10.1109/tbme.2007.908310.

12. Snow RW, Taylor CP, Dudek FE. Electrophysiological and optical changes in slices of rat hippocampus during spreading depression. J Neurophysiol. 1983;50(3):561. https://doi.org/10.1152/jn.1983.50.3.561.

13. Yaari Y, Konnerth A, Heinemann U. Nonsynaptic epileptogenesis in the mammalian hippocampus in vitro. II. Role of extracellular potassium. J Neurophysiol. 1986;56(2):424-38. https://doi.org/10.1152/jn.1986.56.2.424.

14. Herreras O, Largo C, Ibarz JM, Somjen GG, Martin DRR. Role of neuronal synchronizing mechanisms in the propagation of spreading depression in the in vivo hippocampus. J Neurosci. 1994;14(11):7087-98. https://doi.org/10.1523/ jneurosci.14-11-07087.1994.

15. Holsheimer J, Demeulemeester H, Nuttin B, de Sutter P. Identification of the target neuronal elements in electrical deep brain stimulation. Eur J Neurosci. 2000;12(12):4573-7. https://doi.org/10.1111/j.1460-9568.2000.01306.x.

16. Nowak LG, Bullier J. Axons, but not cell bodies, are activated by electrical stimulation in cortical gray matter. II. Evidence from selective inactivation of cell bodies and axon initial segments. Exp Brain Res. 1998;118(4):489-500. https ://doi.org/10.1007/s002210050305.

17. Kloosterman F, Peloquin P, Leung LS. Apical and basal orthodromic population spikes in hippocampal CA1 in vivo show different origins and patterns of propagation. J Neurophysiol. 2001;86(5):2435-44. https://doi.org/10.1152/ jn.2001.86.5.2435.

18. Bragin A, Penttonen M, Buzsáki G. Termination of epileptic afterdischarge in the hippocampus. J Neurosci. 1997;17(7):2567-79. https://doi.org/10.1523/jneurosci.17-07-02567.1997.

19. Dreier JP. The role of spreading depression, spreading depolarization and spreading ischemia in neurological disease. Nat Med. 2011;17(4):439-47. https://doi.org/10.1038/nm.2333.

20. Feng $Z$, Zheng $X, Y u Y$, Durand DM. Functional disconnection of axonal fibers generated by high frequency stimulation in the hippocampal CA1 region in-vivo. Brain Res. 2013;1509:32-42. https://doi.org/10.1016/j.brain res.2013.02.048.

21. Bures J. Leão's spreading depression of EEG activity. Brain Res Bull. 1999;50(5-6):459. https://doi.org/10.1016/S0361 -9230(99)00133-1. 


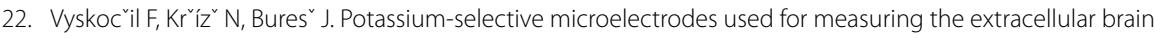
potassium during spreading depression and anoxic depolarization in rats. Brain Res. 1972;39(1):255-9. https://doi. org/10.1016/0006-8993(72)90802-5.

23. Haglund MM, Schwartzkroin PA. Role of Na-K pump potassium regulation and IPSPs in seizures and spreading depression in immature rabbit hippocampal slices. J Neurophysiol. 1990;63(2):225-39. https://doi.org/10.1152/ jn.1990.63.2.225.

24. Traynelis SF, Dingledine R. Potassium-induced spontaneous electrographic seizures in the rat hippocampal slice. J Neurophysiol. 1988;59(1):259-76. https://doi.org/10.1152/jn.1988.59.1.259.

25. Moddel G, Gorji A, Speckmann EJ. Background potassium concentrations and epileptiform discharges. I. Electrophysiological characteristics of neuronal activity. Brain Res. 2003;959(1):135-48. https://doi.org/10.1016/s0006 -8993(02)03741-1.

26. Lian XY, Stringer JL. Astrocytes contribute to regulation of extracellular calcium and potassium in the rat cerebral cortex during spreading depression. Brain Res. 2004;1012(1-2):177-84. https://doi.org/10.1016/j.brain res.2004.04.011.

27. Somjen GG. Mechanisms of spreading depression and hypoxic spreading depression-like depolarization. Physiol Rev. 2001;81(3):1065-96. https://doi.org/10.1152/physrev.2001.81.3.1065.

28. Larrosa B, Pastor J, López-Aguado L, Herreras O. A role for glutamate and glia in the fast network oscillations preceding spreading depression. Neuroscience. 2006;141(2):1057-68. https://doi.org/10.1016/j.neuroscience.2006.04.005.

29. Zhou N, Rungta RL, Malik A, Han H, Wu DC, MacVicar BA. Regenerative glutamate release by presynaptic NMDA receptors contributes to spreading depression. J Cereb Blood Flow Metab. 2013;33(10):1582-94. https://doi. org/10.1038/jcbfm.2013.113.

30. Billups B, Attwell D. Modulation of non-vesicular glutamate release by pH. Nature. 1996;379(6561):171-4. https://doi. org/10.1038/379171a0.

31. Florence CM, Baillie LD, Mulligan SJ. Dynamic volume changes in astrocytes are an intrinsic phenomenon mediated by bicarbonate ion flux. PLoS ONE. 2012;7(11):e51124. https://doi.org/10.1371/journal.pone.0051124.

32. Brummer SB, Turner MJ. Electrochemical considerations for safe electrical stimulation of the nervous system with platinum electrodes. IEEE Trans Biomed Eng. 1977;24(1):59-63. https://doi.org/10.1109/TBME.1977.326218.

33. Morton SL, Daroux ML, Mortimer JT. The role of oxygen reduction in electrical stimulation of neural tissue. J Electrochem Soc. 1994;141(1):122-30. https://doi.org/10.1149/1.2054671.

34. Feng Z, Yu Y, Guo Z, Cao J, Durand DM. High frequency stimulation extends the refractory period and generates axonal block in the rat hippocampus. Brain Stimul. 2014;7(5):680-9. https://doi.org/10.1016/j.brs.2014.03.011.

35. Yu Y, Feng Z, Cao J, Guo Z, Wang Z, Hu N, et al. Modulation of local field potentials by high-frequency stimulation of afferent axons in the hippocampal CA1 region. J Integr Neurosci. 2016;15(01):1-17. https://doi.org/10.1142/s0219 635216500011.

\section{Publisher's Note}

Springer Nature remains neutral with regard to jurisdictional claims in published maps and institutional affiliations. 